\title{
Production of hypernuclei in multifragmentation of nuclear spectator matter
}

\author{
A.S. Botvina ${ }^{1}$ and J. Pochodzalla ${ }^{2}$ \\ ${ }^{1}$ Institute for Nuclear Research, Russian Academy of Sciences, 117312 Moscow, Russia \\ ${ }^{2}$ Institut für Kernphysik, Johannes Gutenberg-University, Mainz, D-55099 Germany
}

(Dated: October 24, 2018)

\begin{abstract}
In peripheral collisions of relativistic heavy ions highly excited spectators containing $\Lambda$ hyperons can be produced. Such strange spectator matter may undergo a break-up into many fragments (multifragmentation) as it is well established for ordinary nuclear systems. We generalize the statistical multifragmentation model, previously successfully used for the description of experimental data, for the case of hypernuclear systems. We predict relative yields of hypernuclei and the main characteristics of such a break-up. We point at a connection of this phenomenon with a liquid-gas phase transition in hypermatter.
\end{abstract}

PACS numbers: $25.70 . \mathrm{Pq}, 21.80 .+\mathrm{a}, 21.65 .+\mathrm{f}$

One promising way to study hypernuclei is to use the copious production of hyperons in relativistic heavy ion collisions. As seen in experiments in the GeV range [1], $\Lambda$ hyperons originating from the hot participant region have a broad rapidity distribution and a considerable fraction can be found in the spectator kinematical region. In peripheral collisions these $\Lambda$ hyperons may be captured by excited spectators. Subsequently these spectators may decay producing cold and possibly exotic hypernuclei. It has been demonstrated experimentally that the cross section of this reaction may be on the order of a few microbarns [2]. A similar value can be obtained with UrQMD calculations by assuming a coalescence of the produced $\Lambda$ hyperons with spectators [3]. Indeed there are extensive plans to investigate hypernuclei produced in such heavy ion reactions using stable [2] as well as exotic beams [3].

An important feature of these reactions is a correlation between the excitation energy transferred to the spectator matter and the number of high energy particles produced as a result of collisions between nucleons in the participant region (see e.g. [4]), which are also related to the strangeness production. As a consequence the excited spectators break-up into many fragments (multifragmentation) [5, 6, , 7], and the captured $\Lambda$ will be finally attached to these fragments. According to the present understanding, multifragmentation is a relatively fast process, with a characteristic time around 100 $\mathrm{fm} / \mathrm{c}$, where, nevertheless, a high degree of equilibration (chemical equilibrium) is reached [8]. This is a result of the strong interaction between the nucleons, and we believe that a small admixture of other particles like hyperons interacting with similar cross sections [9] will not change the general statistical behaviour. Multifragmentation can also be associated with the liquid-gas phase transition in nuclear systems, and thermodynamical features of this transition were experimentally investigated [10]. As was demonstrated by numerous comparisons of theory and experiment, statistical models allow a successful description of experimental data [5, 7, 11, 12]. In this letter we demonstrate that multifragmentation of hypermatter can be described in a similar way, and some properties of hypernuclei can be explored by exam- ining relative yields of hyperfragments. The calculations also show that the usage of exotic heavy ion beams will indeed allow to produce hypernuclei with extreme $\mathrm{N} / \mathrm{Z}$ ratios and thus hypernuclei which are not experimentally accessible with any other technique.

For the theoretical description of this process we modify the grand-canonical version of the Statistical Multifragmentation Model (SMM) [5, 13]. Previously, this version, with explicit addition of mass, charge, energy and momentum conservations, was successfully used for the description of multifragmentation in relativistic heavyion reactions 7, 11, 12. Microcanonical extensions of statistical models, like [14], are essential only for a few details of the phase transition, but not for the general description of the produced fragments. The model assumes that a hot nuclear spectator with total mass (baryon) number $A_{0}$, charge $Z_{0}$, number of $\Lambda$ hyperons $H_{0}$, and temperature $T$ expands to a low density freeze-out volume, where the system is in chemical equilibrium. The statistical ensemble includes all break-up channels composed of nucleons and excited fragments. The primary fragments are formed in the freeze-out volume $V$. We use the excluded volume approximation $V=V_{0}+V_{f}$, where $V_{0}=A_{0} / \rho_{0} \quad\left(\rho_{0} \approx 0.15 \mathrm{fm}^{-3}\right.$ is the normal nuclear density), and parametrize the free volume $V_{f}=\kappa V_{0}$, with $\kappa \approx 2$ [7, 11, 12].

Nuclear clusters in the freeze-out volume are described as follows: light fragments with mass number $A<4$ are treated as elementary particles with corresponding spin and translational degrees of freedom ("nuclear gas"). Their binding energies were taken from experimental data [5, 15, 16]. Fragments with $A=4$ are also treated as gas particles with table masses, however, some excitation energy is allowed $E_{x}=A T^{2} / \varepsilon_{0}\left(\varepsilon_{0} \approx 16 \mathrm{MeV}\right.$ is the inverse volume level density parameter [5]), that reflects the presence of excited states in ${ }^{4} \mathrm{He},{ }_{\Lambda}^{4} \mathrm{H}$, and ${ }_{\Lambda}^{4} \mathrm{He}$ nuclei. Fragments with $A>4$ are treated as heated liquid drops. In this way one can study the nuclear liquid-gas coexistence of hypermatter in the freeze-out volume. The internal free energies of these fragments are parametrized as the sum of the bulk $\left(F_{A}^{B}\right)$, the surface $\left(F_{A}^{S}\right)$, the symmetry $\left(F_{A Z H}^{\mathrm{sym}}\right)$, the Coulomb $\left(F_{A Z}^{C}\right)$, and the hyper energy 
$\left(F_{A H}^{\mathrm{hyp}}\right)$ :

$$
F_{A Z H}(T, V)=F_{A}^{B}+F_{A}^{S}+F_{A Z H}^{\mathrm{sym}}+F_{A Z}^{C}+F_{A H}^{\mathrm{hyp}} .
$$

Here, $H$ denotes the number of $\Lambda$ 's. The first three terms are written in the standard liquid-drop form [5]: $F_{A}^{B}(T)=\left(-w_{0}-\frac{T^{2}}{\varepsilon_{0}}\right) A, \quad F_{A}^{S}(T)=\beta_{0}\left(\frac{T_{c}^{2}-T^{2}}{T_{c}^{2}+T^{2}}\right)^{5 / 4} A^{2 / 3}$, and $F_{A Z H}^{\mathrm{sym}}=\gamma \cdot(A-H-2 Z)^{2} /(A-H)$. The model parameters $w_{0}=16 \mathrm{MeV}, \beta_{0}=18 \mathrm{MeV}, T_{c}=18 \mathrm{MeV}$ and $\gamma=25 \mathrm{MeV}$ were extracted from nuclear phenomenology and provide a good description of multifragmentation data [5, 7, 11, 12]. The Coulomb interaction of the fragments is described within the Wigner-Seitz approximation, and $F_{A Z}^{C}$ is taken as in ref. [5].

The new term is the free hyper-energy $F_{A H}^{\text {hyp }}$. We assume that it does not change with temperature, i.e., it is determined solely by the binding energy of the hyperfragments. Presently, only few ten masses of single hypernuclei (mostly light ones) are experimentally established [15, 16], and there is only very limited information on double hypernuclei available. However, there are some theoretical estimations of their masses based on a description of the available data. One of them is the Samanta formula [17] which suggests a hyper term

$$
E_{\text {sam }}^{\text {hyp }}=H \cdot\left(-10.68+48.7 /\left(A^{2 / 3}\right)\right) .
$$

The contribution proportional to $A^{-2 / 3}$ is motivated by calculations of the $\Lambda$ binding energy in a potential well [18]. In what later follows we use $F_{A H}^{\text {hyp }}=E_{s a m}^{\text {hyp }}$ as one of the versions for our calculations. We have also explored another hyper term (in the following we call it the liquid drop hyper term):

$$
F_{A H}^{\text {hyp }}=(H / A) \cdot\left(-10.68 A+21.27 A^{2 / 3}\right) .
$$

In this formula the binding energy is proportional to the fraction of hyperons in the system $(H / A)$. The second part represents the volume contribution reduced by the surface term and thus resembles a liquid-drop parametrization based on the saturation of the nuclear interaction. The linear dependence at small $H / A$ is in agreement with theoretical predictions [19] for hyper matter. We have found that for single hypernuclei with $A>8$ the average deviation of the liquid-drop masses from the experimental masses tabulated in [15] is 1.8 $\mathrm{MeV}$, while using the Samanta formula this deviation is worse and amounts to $2.2 \mathrm{MeV}$. If not mentioned otherwise we use the liquid-drop hyper term in the following.

At this point we should note that the properties of the primary fragments may change in the medium compared to the vacuum due to the proximity of other fragments. Presently, there are evidences that the symmetry energy [20, 21, 22] and surface energy [23] of hot fragments in multifragmentation may be modified. However, these corrections are not large and influence multifragmentation in finite nuclear systems very little. They are more important for astrophysical applications [24]. Moreover,

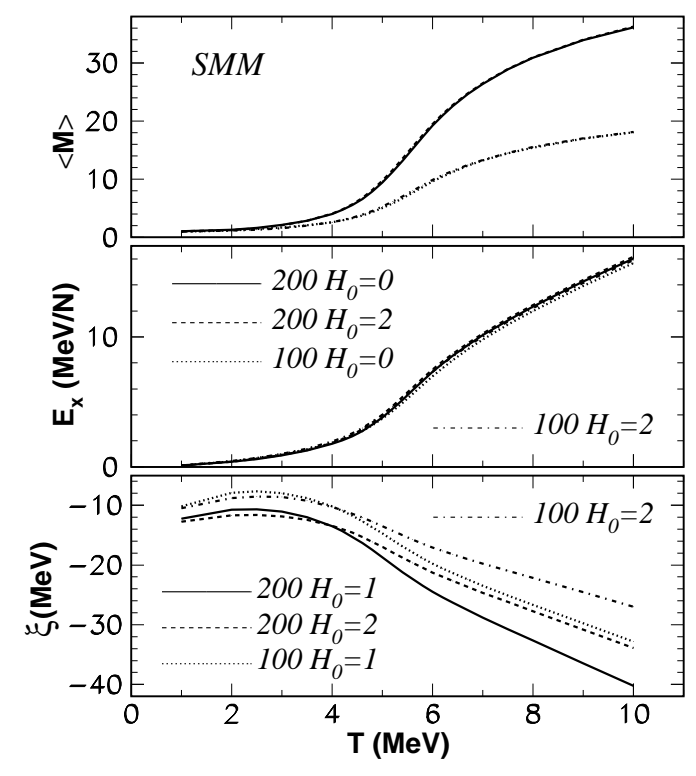

FIG. 1: SMM calculations of multifragmentation of spectator sources with $A_{0}=200, Z_{0}=80$ and $A_{0}=100, Z_{0}=40$, with number of $\Lambda$ hyperons $H_{0}=0,1,2$. Top panel - average multiplicity of produced primary fragments (notations are as in the middle panel), middle panel - excitation energy of the sources (caloric curve), bottom panel - chemical potential $\xi$ responsible for strangeness, as function of temperature $T$.

since the binding energies of hypernuclei are mostly not known even in vacuum, this problem is naturally included in searching for a reliable mass formula for hypernuclei within this approach.

The break-up channels should be selected according to their statistical weight. In the Grand Canonics this leads to the following average yields of individual fragments:

$$
\begin{array}{r}
Y_{\mathrm{AZH}}=g_{\mathrm{AZH}} V_{f} \frac{A^{3 / 2}}{\lambda_{T}^{3}} \exp \left[-\frac{1}{T}\left(F_{A Z H}-\mu_{A Z H}\right)\right], \\
\mu_{A Z H}=A \mu+Z \nu+H \xi,
\end{array}
$$

Here $g_{\mathrm{AzH}}$ is the ground-state degeneracy factor of species $(A, Z, H), \lambda_{T}=\left(2 \pi \hbar^{2} / m_{N} T\right)^{1 / 2}$ is the nucleon thermal wavelength, $m_{N} \approx 939 \mathrm{MeV}$ is the average nucleon mass. In our case $H<<A$, therefore, the mass difference between nucleons and $\Lambda$ can be disregarded in this expression. The chemical potentials $\mu, \nu$, and $\xi$ are responsible for the mass (baryon) number, charge, and strangeness conservation in the system. They can be found from the balance equations:

$$
\sum_{A Z H} A Y_{\mathrm{AZH}}=A_{0}, \sum_{A Z H} Z Y_{\mathrm{AZH}}=Z_{0}, \sum_{A Z H} H Y_{\mathrm{AZH}}=H_{0} .
$$

Within this extended SMM approach we have performed calculations for excited spectator sources which can be produced during peripheral relativistic heavy ion collisions. Below, we show results for heavy systems with $A_{0}=200$ and $Z_{0}=80$, and intermediate systems with 


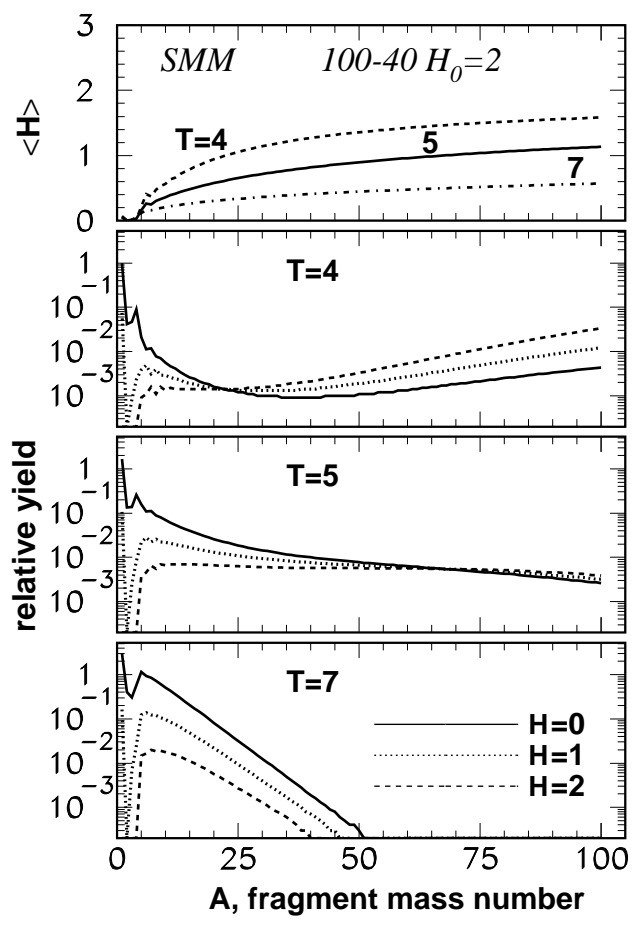

FIG. 2: Multifragmentation of sources with $A_{0}=100, Z_{0}=40$, $H_{0}=2$. Top panel - average number of hyperons in fragments at temperatures $T=4,5$, and $7 \mathrm{MeV}$. Three bottom panels relative yields of fragments (per event), separately for fragments containing $H=0,1$, or $2 \Lambda$, at the same temperatures.

$A_{0}=100$ and $A_{0}=50$, varying the charge-to-mass ratio $(Z / A)$ from 0.4 to 0.5 , and $H_{0}$ from 0 to 2 . The first source has the mass and $Z / A$ ratio typical for a heavy projectile. We expect the most extensive absorption of $\Lambda$ hyperons by spectators in the case of heavy projectiles and targets. It will be possible to increase the strangeness production by selecting smaller impact parameters, leading to a higher excitation and a smaller mass of the projectile spectators. However, previous analyses [20] suggest that their $Z / A$ ratio will be nearly conserved.

In Fig. 11 we present the average multiplicity of fragments produced after the break-up of the excited spectators, the caloric curve for these break-ups, and the chemical potential $\xi$, versus the temperature of the spectator matter. At small temperatures $(T<3 \mathrm{MeV})$, the compound nucleus channel $(M=1)$ dominates. In the following it should decay by means of evaporation or fission processes [5]. One can consider it as a nuclear liquid phase. At temperatures $T=4-6 \mathrm{MeV}$ the system produces mainly several intermediate mass fragments (IMF, with $4<A \lesssim A_{0} / 3$ ), however, a heavy residue and light particles are also possible. In nuclear multifragmentation this region is interpreted as a liquid-gas phase coexistence [5, 10]. Here the caloric curve signals an increasing heat capacity, associated with a rapid rise of the excitation energy. And at high excitations $(T>8-9 \mathrm{MeV})$ the system disintegrates mainly into light particles (a gas phase). One can see that the small admixture of $\Lambda$ 's does
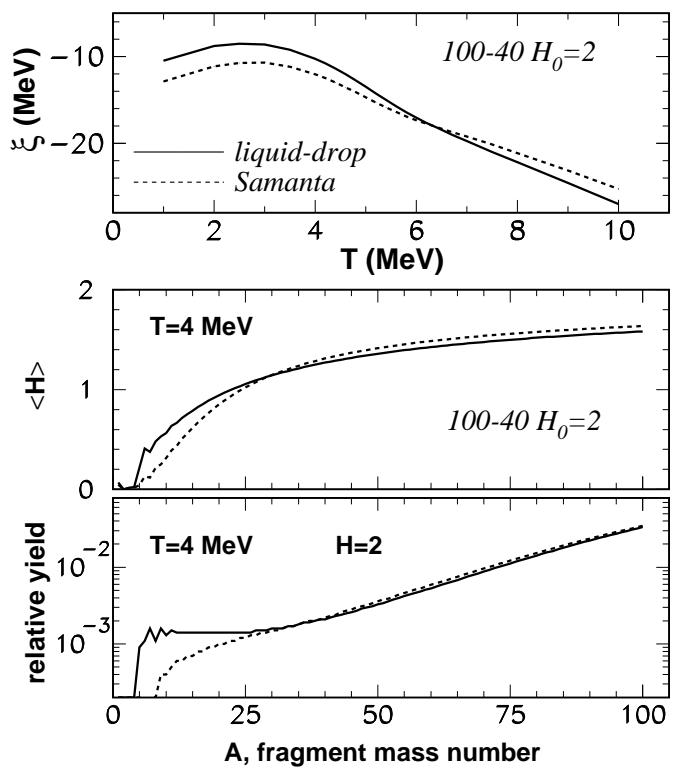

FIG. 3: Comparison of SMM calculations with the liquid-drop and Samanta descriptions of hyper terms in the mass formula, for the same sources as in Fig. 2. Top panel - the strangeness chemical potential $\xi$ versus temperature $T$. Middle panel average number of $\Lambda$ hyperons in fragments, and bottom panel - yields of fragments with two $\Lambda$, at $T=4 \mathrm{MeV}$.

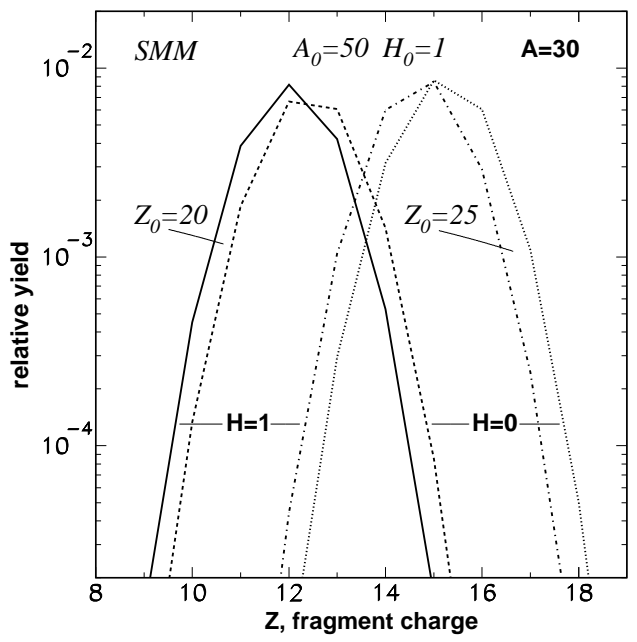

FIG. 4: Charge distributions of primary fragments with $A=30$ produced after break-up of sources with $A_{0}=50, H_{0}=1$, $Z_{0}=20$ (solid and dashed lines), and $Z_{0}=25$ (dotted and dotdashed lines), at temperature $T=5 \mathrm{MeV} . H=0$ stands for fragments without $\Lambda, H=1$ are fragments with one $\Lambda$.

not change this general behaviour. However, a drop of $\xi$ at $T>4 \mathrm{MeV}$ signals a decreasing average strangeness content of the fragments.

In the top panel of Fig. 2 we explicitly demonstrate this decrease of the average number of $\Lambda$ 's contained in the fragments with rising temperature. In three bottom panels we show how the mass distributions of fragments evolve with the temperature. Around the onset of mul- 
tifragmentation $(T \approx 4 \mathrm{MeV})$ there is a "U-shaped" distribution with a big residue and few small fragments. The residue contains nearly all strangeness of the system, while the small fragments are mainly without hyperons. Also the share of free $\Lambda$ 's is not essential, hyperons prefer to be bound in fragments in all cases. When the system breaks into many fragments, the strangeness is distributed among all produced IMF. Within the phase coexistence region $(T \approx 5 \mathrm{MeV})$ we have a rather flat distributions for the produced big residues, and nearly equal probabilities for producing the residues with different number of hyperons. Large fluctuations of number of fragments and their size are expected in this case.

We have found that there is a sensitivity of the fragment yields in multifragmentation to the mass formulae used for description of binding energy of hypernuclei. In Fig. 3. we compare SMM calculations performed with the liquid-drop hyper term (3) in free energy of individual fragments, and with the Samanta term (2). There is a clear difference in the chemical potential $\xi$, and, as a result, the yields of hyper-fragments are also different. As one can see from the bottom panels the liquiddrop formula predicts more strangeness in IMF's than the Samanta formula. The difference in the yields is particularly large for small double hyper-fragments. In future, this observable may allow to test experimentally different mass formulas for hypernuclei in multifragmentation.

It is an important feature that the isotope composition of the produced hypernuclei can be varied considerably in these reactions. In Fig. 4 we show charge distributions of fragments with $\mathrm{A}=30$ after the break-up of spectator sources with different isospin. The corresponding $Z_{0} / A_{0}$ ratios can be easily obtained by selecting different projectiles. After multifragmentation, the hot primary fragments have approximately the same average isospin content as the sources, whereas the widths of the charge distributions depend mainly on the fragment symmetry energy [14]. Thus the present work shows that it is indeed possible to produce very neutron rich hyper-fragments. We expect that the final isospin distributions will be similar to the ones reported in previous multifragmentation studies with exotic beams [8].

After the break-up, the Coulomb acceleration and the secondary de-excitation of primary hot fragments should be taken into account [5]. As it is well known from calculations and experiments, the de-excitation of nuclei with $A \leq 200$ will proceed mainly by emission of nucleons. As a consequence the resulting distributions of cold nuclei are not very different from the primary ones, they are just shifted to lower masses. In this case the reported regularities will not change, and the differences in relative yields will survive, though some of them may become smaller [5, 21, 22]. The last stage will be the mesonic or non-mesonic decay of hypernuclei which can used for the identification of the hypernuclei [3].

We conclude that multifragmentation reactions offer a new possibility for investigating hypernuclei under conditions essentially different from those accessible in conventional nuclear structure studies. One of the advantages is that in this reaction one can abundantly produce hypernuclei with unusual $Z / A$ ratios. It is encouraging that one can distinguish between different mass formulae of hypernuclei, since their properties are manifested in their relative yields. More generally, we suggest that this reaction can be considered as an experimental tool to study clusterization of nuclear matter with strangeness, and the phase diagram of hyper-nuclear matter at densities $\rho \approx 0.1-0.3 \rho_{0}$ and temperatures around $T \approx 3-8$ $\mathrm{MeV}$ reached in the freeze-out volume. Apparently, such a phase transition may take place in some astrophysical cases, for example, in neutron star crusts.

The authors thank I.N. Mishustin, T.R. Saito and W. Trautmann for stimulating discussions. This work was partly supported by the Bundesministerium für Bildung, Wissenschaft, Forschung und Technologie, Germany, under contract number 06-MZ-225I.
[1] X. Lopez et al., Prog. Part. Nucl. Phys. 53, 149 (2004).

[2] S. Avramenko et al., Nucl. Phys. A547, 95c (1992).

[3] The HypHI collaboration: Letter of Intent (2005), http://www.gsi.de/documents/DOC-2005-Feb-432-2.pdf and Progress Report (2006), http://www.gsi.de/documents/DOC-2006-Jan-2-1.pdf.

[4] K. Turzo et al., Eur. Phys. J. A21, 293 (2004).

[5] J.P. Bondorf, A.S. Botvina, A.S. Iljinov, I.N. Mishustin and K. Sneppen, Phys. Rep. 257, 133 (1995).

[6] J. Pochodzalla, Prog. Part. Nucl. Phys. 39, 443 (1997).

[7] R.P. Scharenberg et al., Phys. Rev. C64, 054602 (2001).

[8] For a recent review see Eur. Phys. J. A 30, 1-342 (2006).

[9] The Particle Data Group, W.-M. Yao et al., J. Phys. G 33, 1 (2006).

[10] J. Pochodzalla et al., Phys. Rev. Lett. 75, 1040 (1995).

[11] A.S. Botvina et al., Nucl. Phys. A584, 737 (1995).

[12] H. Xi et al., Z. Phys. A359, 397 (1997).

[13] A.S. Botvina, A.S. Iljinov, and I.N. Mishustin, Sov. J.
Nucl. Phys. 42, 712 (1985).

[14] A.S. Botvina and I.N. Mishustin, Phys. Rev. C 63, 061601(R) (2001).

[15] H. Bando, T. Motoba, and J. Zofka, Int. J. Mod. Phys. A5, 4021 (1990).

[16] O. Hashimoto and H.Tamura, Prog. Part. Nucl. Phys. 57, 564 (2006).

[17] C. Samanta, P.R. Chowdhurry, and D.N. Basu, J. Phys. G: Nucl. Part. Phys. 32, 363 (2006).

[18] D. Rote and A. Bodmer, Nucl. Phys. A 148, 97 (1970).

[19] W. Greiner, Int. J. Mod. Phys. E5, 1 (1995).

[20] A. Le Fèvre et al., Phys. Rev. Lett. 94, 162701 (2005).

[21] J. Iglio et al., Phys. Rev. C74, 024605 (2006).

[22] G. Souliotis et al., Phys. Rev. C75, 011601 (2007).

[23] A.S. Botvina et al., Phys. Rev. C74, 044609 (2006).

[24] A.S. Botvina and I.N. Mishustin, Phys. Rev. C72, 048801 (2005). 